TITLE:

\title{
Chemical effect on muonic atom formation through muon transfer reaction in benzene and cyclohexane samples
}

\section{$\operatorname{AUTHOR}(\mathrm{S})$ :}

Inagaki, Makoto; Ninomiya, Kazuhiko; Nambu, Akihiro; Kudo, Takuto; Terada, Kentaro; Sato, Akira; Kawashima, Yoshitaka; Tomono, Dai; Shinohara, Atsushi

\section{CITATION:}

Inagaki, Makoto ... [et al]. Chemical effect on muonic atom formation through muon transfer reaction in benzene and cyclohexane samples. Radiochimica Acta 2021, 109(4): $319-326$

\section{ISSUE DATE:}

2021-04

URL:

http://hdl.handle.net/2433/267511

\section{RIGHT:}

(C) 2021 Walter de Gruyter GmbH, Berlin/Boston; The full-text file will be made open to the public on 11 February 2022 in accordance with publisher's 'Terms and Conditions for Self-Archiving'. 
Makoto Inagaki^, Kazuhiko Ninomiya, Akihiro Nambu, Takuto Kudo, Kentaro Terada, Akira Sato, Yoshitaka Kawashima, Dai Tomono and Atsushi Shinohara

\section{Chemical effect on muonic atom formation through muon transfer reaction in benzene and cyclohexane samples}

https://doi.org/10.1515/ract-2020-0112

Received November 5, 2020; accepted January 26, 2021;

published online February 11, 2021

\begin{abstract}
To investigate the chemical effect on the muon capture process through a muon transfer reaction from a muonic hydrogen atom, the formation rate of muonic carbon atoms is measured for benzene and cyclohexane molecules in liquid samples. The muon transfer rate to carbon atoms of the benzene molecule is higher than that to the carbon atoms of the cyclohexane molecule. Such a deviation has never been observed among those molecules for gas samples. This may be because the transfers occur from the excited states of muonic hydrogen atoms in the liquid system, whereas in the gas system, all the transfers occur from the $1 \mathrm{~s}$ (ground) state of muon hydrogen atoms. The muonic hydrogen atoms in the excited states have a larger radius than those in the $1 s$ state and are therefore considered to be affected by the steric hindrance of the molecular structure. This indicates that the excited states of muonic hydrogen atoms contribute significantly to the chemical effects on the muon transfer reaction.
\end{abstract}

Keywords: chemical effect; muonic atom; muonic X-ray; pionic atom; transfer reaction.

\section{Introduction}

Muons are elementary particles belonging to lepton; their mass is 207 times that of electrons and they have a lifetime of $2.2 \mu \mathrm{s}$. Because muons have the same negative charge as

\footnotetext{
*Corresponding author: Makoto Inagaki, Institute for Integrated Radiation and Nuclear Science, Kyoto University, Kumatori, Sennan, Osaka 590-0494, Japan; and Graduate School of Science, Osaka University, Toyonaka, Osaka 560-0043, Japan,

E-mail: inagaki.makoto.2r@kyoto-u.ac.jp Kazuhiko Ninomiya, Akihiro Nambu, Takuto Kudo, Kentaro Terada, Akira Sato and Atsushi Shinohara, Graduate School of Science, Osaka University, Toyonaka, Osaka 560-0043, Japan

Yoshitaka Kawashima and Dai Tomono, Research Center for Nuclear Physics, Osaka University, Ibaraki, Osaka 567-0047, Japan
}

electrons, they can form atomic orbitals around the nucleus, such as electrons (muonic orbitals). When a muon penetrates and stops in matter, it is captured in the Coulomb field of an atomic nucleus and one of the electrons is replaced by a muon, forming a muonic orbital. Such atomic systems are known as muonic atoms. The captured muon exists at a highly excited level and is immediately de-excited to the muonic $1 s$ (ground) state while emitting Auger electrons or characteristic X-rays (muonic X-rays). Because the mass of a muon is 207 times that of an electron, the radius of a muonic orbital is approximately $1 / 200$ that of an electronic orbital, and the energy of a muonic X-ray is approximately 200 times that of an electronic X-ray. Recently, the application of muonic $\mathrm{X}$-rays to elemental analysis has been actively studied owing to their high-energy characteristics [1-12].

When a muon is captured by a hydrogen atom, the only electron in the hydrogen atom is replaced by a muon, forming a muonic hydrogen atom comprising a muon and a nucleus (proton). Because muonic hydrogen atoms have no electrons, the size of a muonic hydrogen atom is approximately $1 / 200$ th that of a hydrogen atom. Therefore, the nuclear charge of a muonic hydrogen atom is strongly shielded by the muon, and hence a muonic hydrogen atom behaves as a small and electrically neutral particle [13]. Consequently, a muonic hydrogen atom can diffuse through matter without being repelled by the electron cloud and can approach the nucleus of another atom. As a muonic hydrogen atom approaches the nucleus of another $Z \geq 2$ atom, the muon moves from the muonic hydrogen atom to the deeper muonic orbital of the $Z \geq 2$ atom to form a new muonic atom of $Z \geq 2$. This process is known as a muon transfer reaction [14].

Several studies have investigated the formation process of muonic atoms, and the muon capture probability for each atom in a material has been measured. These studies indicated that the muon capture probability is affected by the chemical environment, such as the molecular structure [15-23]. This known as the chemical effect [22]. For example, the muon capture ratios of boron and nitrogen atoms differ between boron nitride with a 
graphite-type structure and that with a diamond-type structure [23]. Similar studies have been performed with pionic atoms [24]. Pionic atoms are an atomic system similar to muonic atoms, in which one negative pion exists in the atomic system. Because the mass of negative pions is similar to that of muons, the formation processes of pionic and muonic atoms are considered very similar $[25,26]$.

Chemical effects on the formation of pionic and muonic atoms in materials containing hydrogen atoms have been studied. Horváth et al. [27] measured the number of pions captured by hydrogen atoms but not transferred to other atoms by irradiating $\mathrm{H}_{2} \mathrm{O}$ and $\mathrm{NH}_{3}$ with pions at different temperature conditions; they discovered that the number of pions increased with temperature in the liquid state and reached a maximum in the supercritical state. Furthermore, they performed detailed experiments using partially deuterated methanol and concluded that the pion capture rate on a hydrogen atom changed with the change in the electron density around the hydrogen atom owing to the change in the strength of the hydrogen bond with temperature [28]. Shinohara et al. [29] reported that the pion capture probability of hydrogen atoms of hydrocarbon molecules varied with the molecular structure. Furthermore, they reported that the pionic X-ray intensity pattern of the Balmer series varied with the molecular structure of hydrocarbon molecules [30]. For condensed systems, they reported the chemical effect on the pion transfer reaction in mixtures of carbon tetrachloride and benzene or cyclohexane [31]. They reported that the pion transfer rate to the carbon atoms of benzene was approximately twice the rate to the carbon atoms of cyclohexane and concluded that this was due to differences in the steric hindrance around the carbon atoms in benzene and cyclohexane molecules.

The muon transfer reaction in mixtures of hydrogen gas and noble gases at various pressures has been studied, and the pressure dependence of the muonic X-ray intensity pattern has been reported [32]. However, it is unclear whether such a pressure dependence is due to chemical effects on the muon transfer reaction or to differences in the muon de-excitation process following the muon transfer reaction by the sample pressure. Moreover, the muon transfer reaction in a mixture of hydrogen gas and carbon monoxide or carbon dioxide has been investigated, and no clear difference was observed between the two samples [33]. In a previous study, we conducted an experiment on muonic atom formation in gas mixtures comprising hydrogen, neon, and gaseous benzene or gaseous cyclohexane to investigate the chemical effect on the muon transfer reaction in the gas system [34]. Consequently, a chemical effect on the muon transfer to benzene or cyclohexane in the gaseous system was not observed, unlike pionic transfer in a condensed system. Hence, in contrast to the pion transfer process, no clear chemical effect on the muon transfer reaction has been reported.

Although a systematic understanding of the chemical effect in the direct capture process is improving, the chemical effect in the transfer process are rarely reported. In particular, muon transfer reactions are more difficult to observe than pion transfer reactions because it is difficult to experimentally determine the number of muons captured by hydrogen atoms. In this study, the chemical effect on the muon transfer reaction was investigated in an experimental system, in which the chemical effect on the pion transfer reaction was observed, i.e., in the liquid mixtures of carbon tetrachloride and benzene or cyclohexane. The number of muons captured by hydrogen atoms cannot be obtained because muonic hydrogen does not emit high-energy decay gamma rays, unlike the pion case. Therefore, we performed precise experiments based on different mixing ratios of liquids and analyzed the chemical effect on the muon transfer reaction using model equations.

\section{Experimental}

The experiment was performed at the Muon Science Innovative Muon Beam Channel (MuSIC) of the Research Center for Nuclear Physics (RCNP), Osaka University. Muon beam irradiation experiments were conducted at the M1 beamline of MuSIC from June 17 to June 18, 2017, and from October 8 to October 9, 2018. A muon beam provided at the MuSIC was generated by a $392 \mathrm{MeV}$ proton bombardment on the carbon target from the ring cyclotron of the RCNP. The momentum of the muon beam used was $50 \mathrm{MeV} / \mathrm{c}$. The experimental setup comprised two plastic scintillators, a degrader, a sample liquid holder, and two high-purity germanium semiconductor (HPGe) detectors. A schematic diagram of the experimental setup is shown in Figure 1. The sample liquid holder was made of aluminum and rectangular in shape, with an inner volume of approximately $25 \mathrm{~mL}$ and having 0.3 -mm-thick windows. The sample in the holder was placed at the beam center and irradiated with a muon beam, and the muonic X-rays emitted as muons stopped in the sample to form muonic atoms were measured using the HPGe detectors. Two plastic scintillators were placed upstream of the sample to detect the timing of the muon incidence. An aluminum plate with a thickness of $0.2 \mathrm{~mm}$ was installed as a degrader to decelerate the incident muons, and their momentum was adjusted to stop the muons at the middle of the sample. The detection efficiency of each HPGe detector was determined using Monte Carlo simulation code EGS5 [35], based on data measured using a standard radiation source. The self-absorption effect of each sample on the detection efficiency was considered in the simulation. The samples used in the experiments are listed in Table 1. The reagents used for the samples were benzene (Sigma-Aldrich, for HPLC, $\geq 99.9 \%$ purity), cyclohexane (Sigma-Aldrich, for HPLC, $\geq 99.9 \%$ purity), and carbon tetrachloride (Wako, Super Special Grade, 99.8\% purity). The spectrum for 


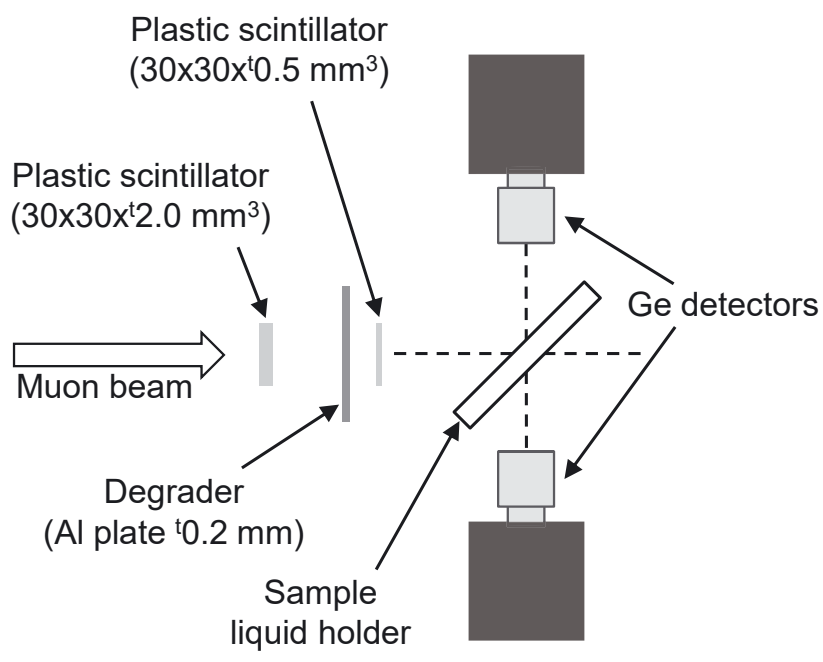

Figure 1: Schematic diagram of experimental setup.

background evaluation was obtained using distilled water. The mixed samples were prepared by mixing while measuring their weights to achieve a predetermined molar ratio. The irradiation times for each sample are listed in Table 1.

\section{Results}

Representative spectra for the $\mathrm{C}_{6} \mathrm{H}_{6}+\mathrm{CCl}_{4}(70 \%)$ sample and $\mathrm{H}_{2} \mathrm{O}$ sample are shown in Figures 2 and 3. The terms $\mu \mathrm{Z}$ $\left(n-n^{\prime}\right)$ and eZ (n- $\left.n^{\prime}\right)$ denote muonic and electronic X-rays resulting from the transition from the principal quantum number $\mathrm{n}$ to $\mathrm{n}^{\prime}$ at the Zth atom, respectively. Muonic X-rays of carbon and chlorine were clearly observed in each

Table 1: List of samples used in the experiments. Compositions are shown in molar percentages. Duration of muon irradiation for each sample is shown as well.

\begin{tabular}{lll}
\hline Sample name & Composition & $\begin{array}{l}\text { Irradiation } \\
\text { time }\end{array}$ \\
\hline $\mathrm{C}_{6} \mathrm{H}_{6}+\mathrm{CCl}_{4}(15 \%)$ & $\mathrm{C}_{6} \mathrm{H}_{6}(85.0 \%)+\mathrm{CCl}_{4}$ & $9 \mathrm{~h} \mathrm{04} \mathrm{min}$ \\
sample & $(15.0 \%)$ & \\
$\mathrm{C}_{6} \mathrm{H}_{6}+\mathrm{CCl}_{4}(30 \%)$ & $\begin{array}{l}\mathrm{C}_{6} \mathrm{H}_{6}(70.0 \%)+\mathrm{CCl}_{4} \\
\text { sample }\end{array}$ & $2 \mathrm{~h} 45 \mathrm{~min}$ \\
$\mathrm{C}_{6} \mathrm{H}_{6}+\mathrm{CCl}_{4}(70 \%)$ & $\mathrm{C}_{6} \mathrm{H}_{6}(30.0 \%)+\mathrm{CCl}_{4}$ & $2 \mathrm{~h} 11 \mathrm{~min}$ \\
sample & $(70.0 \%)$ & \\
$\mathrm{C}_{6} \mathrm{H}_{12}+\mathrm{CCl}_{4}(15 \%)$ & $\mathrm{C}_{6} \mathrm{H}_{12}(85.0 \%)+\mathrm{CCl}_{4}$ & $7 \mathrm{~h} 51 \mathrm{~min}$ \\
sample & $(15.0 \%)$ & \\
$\mathrm{C}_{6} \mathrm{H}_{12}+\mathrm{CCl}_{4}(30 \%)$ & $\mathrm{C}_{6} \mathrm{H}_{12}(70.0 \%)+\mathrm{CCl}_{4}$ & $3 \mathrm{~h} 01 \mathrm{~min}$ \\
sample & $(30.0 \%)$ & \\
$\mathrm{C}_{6} \mathrm{H}_{12}+\mathrm{CCl}_{4}(70 \%)$ & $\mathrm{C}_{6} \mathrm{H}_{12}(30.0 \%)+\mathrm{CCl}_{4}$ & $2 \mathrm{~h} 08 \mathrm{~min}$ \\
sample & $(70.0 \%)$ & \\
$\mathrm{CCl}_{4}$ sample & $\mathrm{CCl}_{4}(100 \%)$ & $1 \mathrm{~h} 30 \mathrm{~min}$ \\
$\mathrm{H}_{2} \mathrm{O}$ sample & Distilled water $(100 \%)_{2} \mathrm{~h} 17 \mathrm{~min}$ \\
\hline
\end{tabular}
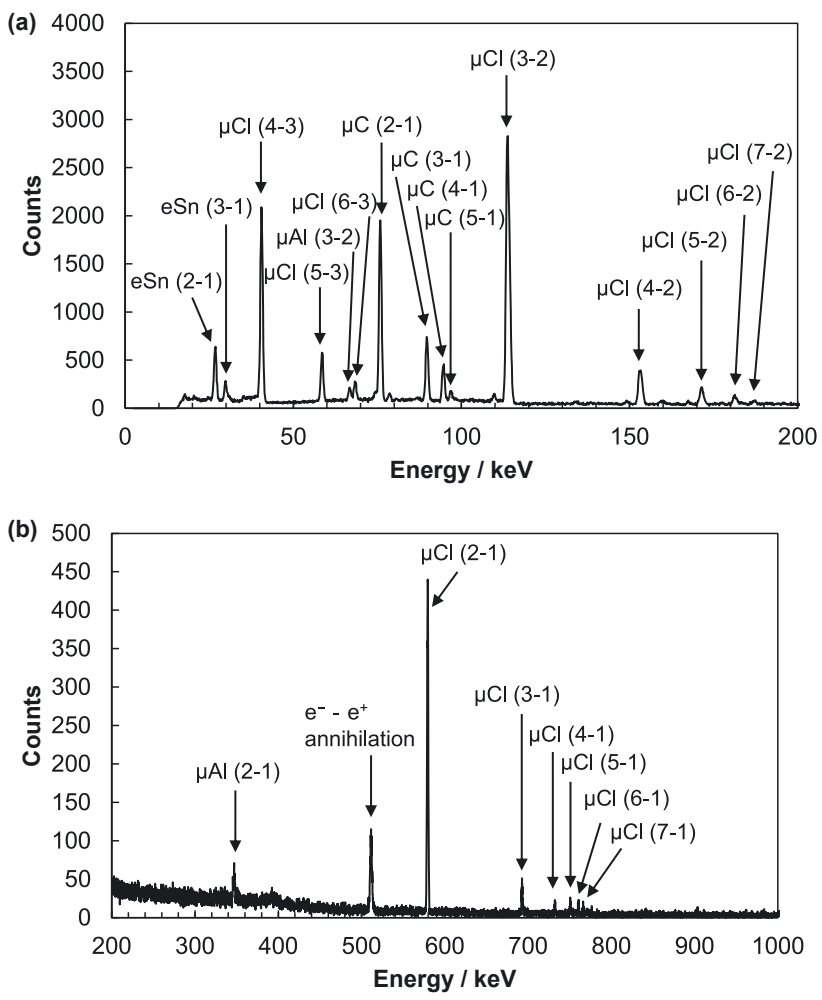

Figure 2: $\mathrm{X}$-ray spectrum for $\mathrm{C}_{6} \mathrm{H}_{6}+\mathrm{CCl}_{4}(70 \%)$ sample. (a) Lowenergy region; (b) high-energy region.

sample. The muonic X-rays of aluminum were from the sample liquid holder, whereas the X-rays of tin were from the tin plate used for shielding the HPGe detectors. In the $\mathrm{H}_{2} \mathrm{O}$ sample for the background evaluation, which did not contain carbon, $\mu \mathrm{Al}$ (4-2) X-rays were observed at the same energy as $\mu \mathrm{C}$ (3-1), although their intensity was low. Therefore, the ratio of $\mu \mathrm{Al}$ (3-2) and $\mu \mathrm{Al}$ (4-2) intensities in the $\mathrm{H}_{2} \mathrm{O}$ sample was used to estimate the intensity of $\mu \mathrm{Al}$ (42 ) in the other samples, and the $\mu \mathrm{C}$ (3-1) intensities were corrected. No other interfering peaks were observed in the muonic X-ray energy range of carbon and chlorine. For each sample, the muonic X-ray intensities of carbon and chlorine were analyzed to obtain the sum of the Lyman series muonic X-rays. Subsequently, the ratio of the Lyman series intensity of carbon to that of chlorine in each sample was determined. The results are shown in Table 2.

\section{Discussion}

\subsection{Muon capture ratio}

Because all muons captured by an atom are finally deexcited to the $1 \mathrm{~s}$ orbital, the total muonic X-ray intensity of 

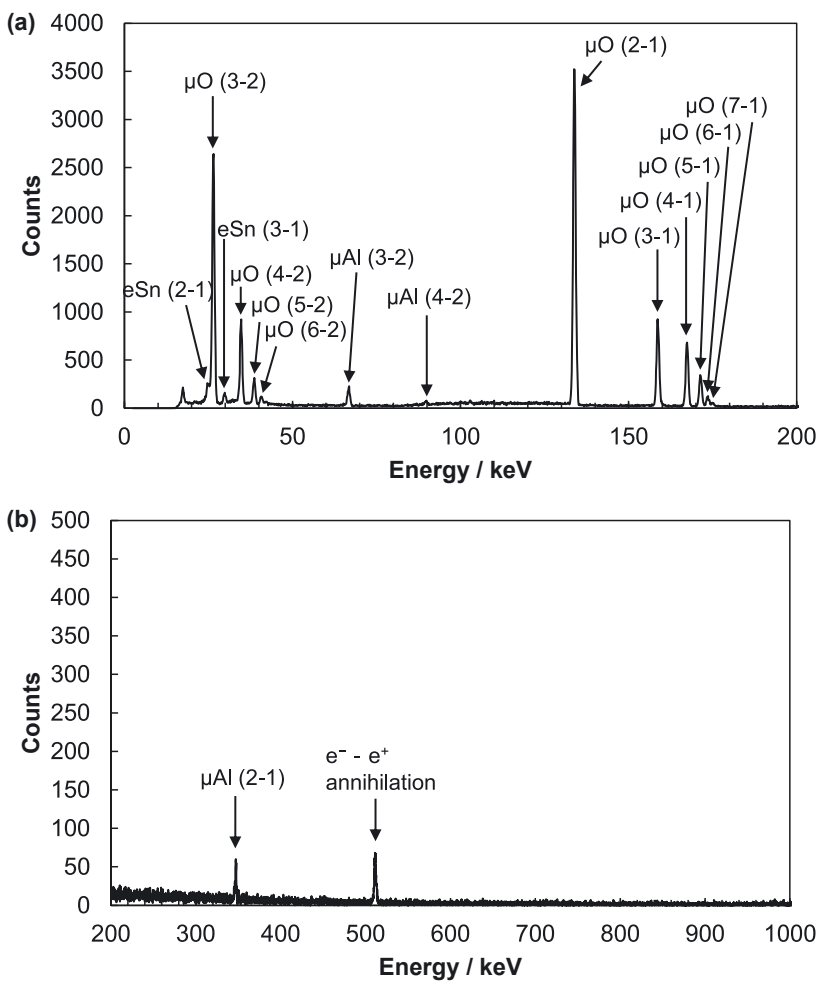

Figure 3: X-ray spectrum for $\mathrm{H}_{2} \mathrm{O}$ sample (background evaluation). (a) Low-energy region; (b) high-energy region.

Table 2: Intensity ratio of total Lyman series muonic X-ray of carbon to that of chlorine in each sample. Intensity fraction of total Lyman series muonic X-ray of carbon to sum of that of carbon and chlorine in each sample is also shown. These ratios and fractions correspond to muon capture ratios and muon capture fractions, respectively.

\begin{tabular}{lrr}
\hline & $\frac{\mu C \text { Lyman }}{\mu C l \text { Lyman }}$ & $\frac{\mu C \text { Lyman }}{\mu C \text { Lyman }+\mu C \text { Lyman }}$ \\
\hline $\mathrm{C}_{6} \mathrm{H}_{6}+\mathrm{CCl}_{4}(15 \%)$ sample & $4.86 \pm 0.14$ & $0.829 \pm 0.004$ \\
$\mathrm{C}_{6} \mathrm{H}_{6}+\mathrm{CCl}_{4}(30 \%)$ sample & $2.00 \pm 0.08$ & $0.667 \pm 0.009$ \\
$\mathrm{C}_{6} \mathrm{H}_{6}+\mathrm{CCl}_{4}(70 \%)$ sample & $0.432 \pm 0.014$ & $0.302 \pm 0.007$ \\
$\mathrm{C}_{6} \mathrm{H}_{12}+\mathrm{CCl}_{4}(15 \%)$ sample & $5.42 \pm 0.16$ & $0.844 \pm 0.004$ \\
$\mathrm{C}_{6} \mathrm{H}_{12}+\mathrm{CCl}_{4}(30 \%)$ sample & $2.27 \pm 0.09$ & $0.694 \pm 0.008$ \\
$\mathrm{C}_{6} \mathrm{H}_{12}+\mathrm{CCl}_{4}(70 \%)$ sample & $0.455 \pm 0.015$ & $0.313 \pm 0.007$ \\
$\mathrm{CCl}_{4}$ sample & $0.073 \pm 0.004$ & $0.068 \pm 0.003$ \\
\hline
\end{tabular}

the Lyman series, i.e., the series leading to the $1 s$ orbital, is proportional to the number of captured muons to each element. Therefore, the ratio of muonic X-ray intensities of the Lyman series of carbon to those of chlorine corresponds to the ratio of the number of muons captured by carbon atoms to that captured by chlorine atoms. The muon capture ratios of carbon atoms to chlorine atoms decreases with the concentration of carbon tetrachloride. In addition, the ratios for the cyclohexane samples are larger than those for the benzene samples with the same concentration of carbon tetrachloride. This indicates that the capture probability is larger for the carbon atoms of cyclohexane than that for the carbon atoms of benzene. However, because this is the result of the sum of the direct muon capture and the capture by muon transfer, further analysis is required to obtain information regarding the muon transfer rate.

\subsection{Analysis of muon transfer rate using model equations}

To determine the muon transfer rate from the capture ratio with different molecular compositions, the following model was constructed. Considering a two-step process in which the muon is first captured by the molecule and subsequently captured by each atom within the molecule, the capture probabilities for the carbon and chlorine atoms ( $W_{\mathrm{C}}$ and $W_{\mathrm{Cl}}$, respectively) are expressed as follows:

$$
\begin{aligned}
W_{\mathrm{C}}= & W_{\mathrm{C}}^{\prime}+W_{\mathrm{H}}^{\prime} \times \frac{\Lambda_{\mathrm{C}} C_{\mathrm{C}}}{\Lambda_{\mathrm{C}} C_{\mathrm{C}}+\Lambda_{\mathrm{Cl}} C_{\mathrm{Cl}}} \\
W_{\mathrm{Cl}}= & W_{\mathrm{Cl}}^{\prime}+W_{\mathrm{H}}^{\prime} \times \frac{\Lambda_{\mathrm{Cl}} C_{\mathrm{Cl}}}{\Lambda_{\mathrm{C}} C_{\mathrm{C}}+\Lambda_{\mathrm{Cl}} C_{\mathrm{Cl}}} \\
W_{\mathrm{C}}^{\prime}= & \frac{K_{\mathrm{HC}}(1-C)}{K_{\mathrm{HC}}(1-C)+K_{\mathrm{CCl}_{4} C} C} \times\left(1-K_{\mathrm{H}}\right) \\
& +\frac{K_{\mathrm{Cll}_{4}} C}{K_{\mathrm{HC}}(1-C)+K_{\mathrm{CCl} l_{4}} C} \times\left(1-K_{\mathrm{Cl}}\right) \\
W_{\mathrm{Cl}}^{\prime}= & \frac{K_{\mathrm{CCl} l_{4}} C}{K_{\mathrm{HC}}(1-C)+K_{\mathrm{Cl}_{4} C} C} \times K_{\mathrm{Cl}} \\
W_{\mathrm{H}}^{\prime}= & \frac{K_{\mathrm{HC}}(1-C)}{K_{\mathrm{HC}}(1-C)+K_{\mathrm{Cll}_{4} C} C} \times K_{\mathrm{H}},
\end{aligned}
$$

where $K_{\mathrm{HC}}$ : capture probability of hydrocarbon molecule; $\mathrm{K}_{\mathrm{CCl}_{4}}$ : capture probability of carbon tetrachloride molecule; $C$ : number of carbon tetrachloride molecules/total number of molecules (= fraction of carbon tetrachloride); $K_{\mathrm{H}}$ : capture probability of hydrogen atoms when muon is captured by hydrocarbon molecules; $K_{\mathrm{Cl}}$ : capture probability of chlorine atom when muon is captured by carbon tetrachloride molecule; $\Lambda_{\mathrm{Z}}$ : transfer rate to $\mathrm{Z}$ atom; $C_{\mathrm{Z}}$ : number of $Z$ atoms per $1 \mathrm{~cm}^{3}$.

The first terms of the $W_{\mathrm{C}}$ and $W_{\mathrm{Cl}}$ equations are the contributions from the direct muon capture, whereas the second terms are the contributions from the muon transfer. $W_{\mathrm{C}}^{\prime}, W_{\mathrm{Cl}}^{\prime}$, and $W_{\mathrm{H}}^{\prime}$ are the direct muon capture probabilities for each atom. The first term of the $W_{\mathrm{C}}^{\prime}$ equation is the contribution of hydrocarbon molecules, whereas the second term is the contribution of carbon tetrachloride 
molecules. The capture probability for chlorine atoms when the muon is captured by a carbon tetrachloride molecule $\left(K_{\mathrm{Cl}}\right)$ was determined from the muon capture ratio of carbon atoms to chlorine atoms in the $\mathrm{CCl}_{4}$ sample. The ratio of capture probabilities of hydrocarbon and carbon tetrachloride molecules $\left(K_{\mathrm{HC}} / K_{\mathrm{CCl}_{4}}\right)$ as well as the capture probability of hydrogen atoms when the muon was captured by hydrocarbon molecules $\left(K_{\mathrm{H}}\right)$ could not be obtained from this experiment; hence, the values obtained from the pion experimental result with the same system and a large mesomolecular (LMM) model were used $[29,36]$. This is based on the assumption that the initial capture process for muons and pions are identical. In the LMM model, $K_{\mathrm{H}}$ is expressed as follows [29]:

$$
K_{\mathrm{H}}=\frac{v(1+\sigma)}{(N+v)(1-\sigma) Z_{\mathrm{eff}}}
$$

Here, $N$ is the sum of the valence electrons for the $\mathrm{C}-\mathrm{C}$ bond and the core electrons other than the $1 s$ electrons in the molecule; $v$ is the sum of the valence electrons associated with the $\mathrm{C}-\mathrm{H}$ bond in the molecule; $Z_{\mathrm{eff}}$ is the sum of the valence electrons and core electrons other than the $1 \mathrm{~s}$ electrons in one carbon atom of the molecule; $\sigma$ is the ionicity parameter, which is zero in $\mathrm{C}-\mathrm{H}$ covalent bonds. In benzene molecules, $N=18, v=12, Z_{\text {eff }}=4$, and $\sigma=0$; in cyclohexane molecules, $N=18, v=24, Z_{\text {eff }}=4$, and $\sigma=0$ [29]. Hence, the calculated $K_{\mathrm{H}}$ for each molecule is as shown in Table 3.

Using the model equation, $K_{\mathrm{HC}} / K_{\mathrm{CCl}_{4}}, K_{\mathrm{H}}$, and $K_{\mathrm{Cl}}$ were fixed to the values shown in Table 3 , and $W_{\mathrm{C}} /\left(W_{\mathrm{C}}+W_{\mathrm{Cl}}\right)$ versus $C$ was fitted to the experimental values shown in Table 2 (the muon capture fractions), with $\Lambda_{\mathrm{C}} / \Lambda_{\mathrm{Cl}}$ as a free parameter for both the $\mathrm{C}_{6} \mathrm{H}_{6}+\mathrm{CCl}_{4}$ and $\mathrm{C}_{6} \mathrm{H}_{12}+\mathrm{CCl}_{4}$ systems. The fitted curves are shown in Figure 4. Because $\Lambda_{C}$ is the relative value to $\Lambda_{\mathrm{Cl}}$ in the model equation, the muon transfer rate to the carbon atom is expressed as $\Lambda_{\mathrm{C}} / \Lambda_{\mathrm{Cl}}$. The fitting results show that the muon transfer rate to the carbon atoms of benzene, $\Lambda_{\mathrm{C}}\left(\mathrm{C}_{6} \mathrm{H}_{6}\right) / \Lambda_{\mathrm{Cl}}$, was $0.51 \pm 0.14$, whereas that to the carbon atoms of cyclohexane, $\Lambda_{\mathrm{C}}$ $\left(\mathrm{C}_{6} \mathrm{H}_{12}\right) / \Lambda_{\mathrm{Cl}}$, was $0.36 \pm 0.05$; and their ratio, $\Lambda_{\mathrm{C}}\left(\mathrm{C}_{6} \mathrm{H}_{6}\right) / \Lambda_{\mathrm{C}}$ $\left(\mathrm{C}_{6} \mathrm{H}_{12}\right)$, was $1.4 \pm 0.4$. In other words, the optimum value of $\Lambda_{\mathrm{C}}$ for benzene was larger than that for cyclohexane, and

Table 3: Parameters used for analysis.

\begin{tabular}{lcrl}
\hline & $\mathrm{C}_{6} \mathrm{H}_{6}$ & $\mathrm{C}_{6} \mathrm{H}_{12}$ & Note \\
\hline$K_{\mathrm{HC}} / K_{\mathrm{CCl} / 4}$ & $0.873 \pm 0.012$ & $1.149 \pm 0.016$ & From pion experimental \\
& & & results [36] \\
$K_{\mathrm{H}}$ & $12 / 120$ & $24 / 144$ & LMM model [29] \\
$K_{\mathrm{Cl}}$ & $0.932 \pm 0.003$ & This study \\
\hline
\end{tabular}

(a)

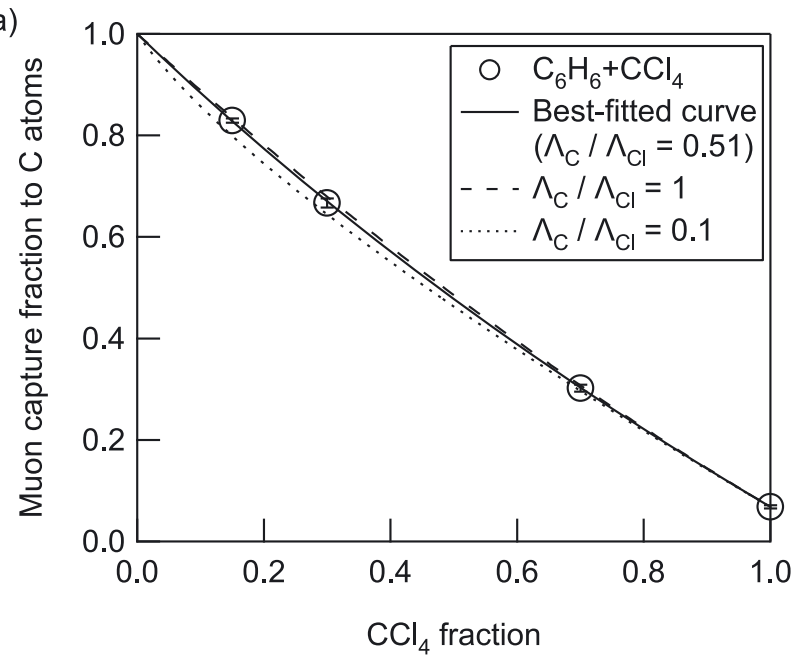

(b)

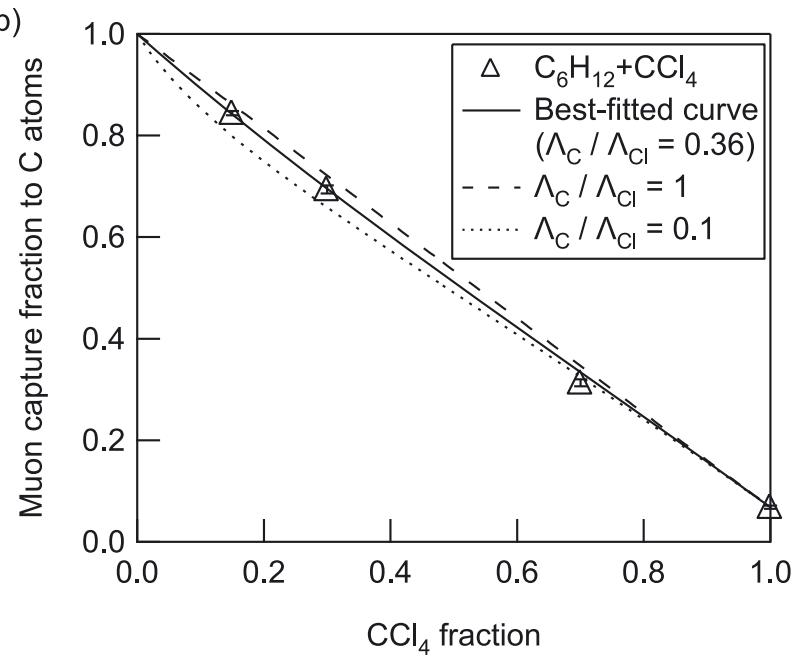

Figure 4: Fitted curves of the muon capture fractions of carbon atoms versus carbon tetrachloride fraction in each sample. Function of $W_{\mathrm{C}} /\left(W_{\mathrm{C}}+W_{\mathrm{C})}\right)$ versus $C$ was fitted to experimental values of muon capture fractions, with $\Lambda_{C} / \Lambda_{C l}$ as a free parameter. The curves for cases where $\Lambda_{C} / \Lambda_{C l}$ is 0.1 or 1 are also shown. (a) For benzene samples; (b) for cyclohexane samples.

$\Lambda_{\mathrm{C}}\left(\mathrm{C}_{6} \mathrm{H}_{6}\right) / \Lambda_{\mathrm{C}}\left(\mathrm{C}_{6} \mathrm{H}_{12}\right)$ was larger than 1 with an accuracy of one sigma.

\subsection{Muon and pion transfer rate in liquid and gas systems}

The muon transfer rates in the liquid system obtained in this study were compared with those in the gas system and liquid system obtained in previous studies. The results are summarized in Table 4.

For the pion transfer in the liquid system, the transfer rate to the carbon atoms of the benzene molecule was approximately twice higher than that to the carbon atoms 
Table 4: Summary of chemical effect on transfer rate in liquid and gas systems.

\begin{tabular}{lrrr}
\hline & $\begin{array}{r}\text { Pion transfer in } \\
\text { the liquid system }\end{array}$ & $\begin{array}{r}\text { Muon transfer in } \\
\text { the liquid system }\end{array}$ & $\begin{array}{r}\text { Muon transfer in } \\
\text { the gas system }\end{array}$ \\
\hline$\Lambda_{\mathrm{C}}\left(\mathrm{C}_{6} \mathrm{H}_{6}\right) /$ & $2.0 \pm 0.4[31]$ & $1.4 \pm 0.4$ & $1.0 \pm 0.1[34]$ \\
$\Lambda_{\mathrm{C}}\left(\mathrm{C}_{6} \mathrm{H}_{12}\right)$ & & & \\
\hline
\end{tabular}

of the cyclohexane molecule [31]. For the muon transfer in the liquid system, the transfer rate to the carbon atoms of the benzene molecule was approximately 1.4 times higher than that to the carbon atoms of the cyclohexane molecule. However, in the muon transfer in the gas system, almost no difference was observed in the transfer rate [34]. In other words, chemical effects were observed in the liquid system but not in the gas system.

These results are attributable to the difference in the proportion of excited states of pionic or muonic hydrogen atoms at the time of transfer. The estimated order of magnitude of the muon transfer rates in the liquid and gas systems are shown in Table 5. The transfer rate to carbon atoms was obtained by converting the literature value of the transfer rate for the $\mathrm{H}_{2}+\mathrm{CH}_{4}$ system of $(9.5 \pm 0.5) \times 10^{10} \mathrm{~s}^{-1}$ [37] while considering the density of carbon atoms for each experiment. The literature value was normalized to $4.25 \times 10^{22} \mathrm{~cm}^{-3}$, which is the atomic density of liquid hydrogen.

In the gas system, the muon transfer rate was on the order of $10^{5} \mathrm{~s}^{-1}$, and the average time required to complete the transfer was on the order of $10^{-6} \mathrm{~s}$. All muonic hydrogen atoms were de-excited to the $1 s$ or $2 s$ state within $10^{-7} \mathrm{~s}$, and the majority of muonic hydrogen atoms were in the $1 s$ state at $10^{-6} \mathrm{~s}$ [38]. Therefore, muon transfers occurred in the gas system after the muon reached the $1 s$ state in the muonic hydrogen atoms. Meanwhile, in the liquid system, the muon transfer rate was on the order of $10^{10} \mathrm{~s}^{-1}$, and the average time required to complete the transfer was on the

Table 5: Muon transfer rates in liquid and gas systems.

\begin{tabular}{lrr}
\hline & $\begin{array}{r}\text { Liquid system } \\
\text { experiment }\end{array}$ & $\begin{array}{r}\text { Gas system } \\
\text { experiment }\end{array}$ \\
\hline $\begin{array}{l}\text { Density of carbon atoms } \\
\text { Muon transfer rate of carbon } \\
\text { atoms }\end{array}$ & $\begin{array}{r}2.7 \times 10^{22} \mathrm{~cm}^{-3} \\
6 \times 10^{10} \mathrm{~s}^{-1}\end{array}$ & $2.9 \times 10^{17} \mathrm{~cm}^{-3}$ \\
Average time for transfer & $2 \times 10^{-11} \mathrm{~s}$ & $2 \times 10^{-6} \mathrm{~s}$ \\
$\begin{array}{l}\text { completion } \\
\begin{array}{l}\text { Percentage of excited } \\
\text { muonic hydrogen }(n \geq 3)\end{array}\end{array}$ & $\sim 70 \%$ & \\
\begin{tabular}{l} 
when transfer occurs [38] \\
\hline
\end{tabular} & & \\
\hline
\end{tabular}

order of $10^{-11} \mathrm{~s}$. At $10^{-11} \mathrm{~s}$ after formation, approximately $70 \%$ of muonic hydrogen atoms were in states with principal quantum numbers $n \geq 3$ [38]. Therefore, in the liquid system, muon transfers occurred mainly from the excited states of muonic hydrogen atoms with principal quantum numbers $n \geq 3$. In the case of pion transfers in liquid systems, pion transfers occurred only from the excited states of the pionic hydrogen atoms because the lifetime of the pionic hydrogen atoms in the $1 s$ state is extremely short $\left(<10^{-15} \mathrm{~s}\right)$ [29].

In the liquid system, the difference in the pion transfer rate to the carbon atoms is believed to be due to the difference in the steric hindrance around the carbon atoms in benzene and cyclohexane molecules [31]. In other words, the hydrogen atoms in the molecules hindered the pionic atoms from approaching the carbon atoms. The steric hindrance to the muonic or pionic hydrogen atom approaching the carbon atom was larger around the cyclohexane molecule than around the benzene molecule because the number of hydrogen atoms bound to each carbon atom in the cyclohexane molecule was two, whereas it was one in the benzene molecule. The radius of the muonic or pionic hydrogen atoms in the excited states was larger than that in the $1 s$ state. For example, the radius of the muonic or pionic hydrogen atom with a principal quantum number $n=3$ was nine times larger than that in the $1 s$ state. Therefore, the higher the proportion of the excited states, the more affected they were by steric hindrance. Consequently, the molecular difference in the transfer rate to carbon atoms was the largest for the pion transfer in the liquid system, in which the transfer occurred only from the excited states. The difference was the smallest for the muon transfer in the gas system, in which no transfer occurred from the excited states. The difference for the muon transfer in the liquid system, in which the transfer from the excited states was approximately $70 \%$, was smaller than that for the pion transfer in the liquid system and larger than that for the muon transfer in the gas system.

\section{Conclusion}

In this study, to investigate the chemical effect on the muon transfer reaction, muon irradiation experiments were performed with benzene and cyclohexane molecules, which have been observed to impose chemical effects on pion transfer.

In the liquid system, the muon transfer rate to the carbon atoms of the benzene molecule was $1.4 \pm 0.4$ times higher than that to the carbon atoms of the cyclohexane 
molecule. This was speculated to be due to the difference in the steric hindrance caused by the molecules, as in the case of pion transfer. The benzene molecule had only one hydrogen atom attached to each carbon atom, whereas the cyclohexane molecule had two hydrogen atoms attached to each carbon atom. Therefore, the cyclohexane molecules posed more obstacles that prevented muonic hydrogen atoms from approaching the carbon atoms compared with the benzene molecules. Therefore, the muon transfer rate to the carbon atoms of the cyclohexane molecules was lower than that to the carbon atoms of the benzene molecules.

In the gas system, almost no difference was observed in the muon transfer rates to the carbon atoms of benzene and cyclohexane molecules [34]. This might be because the transfers occurred from the excited states of the muonic hydrogen atoms in the liquid system, whereas in the gas system, all the transfers occurred from the $1 \mathrm{~s}$ state of the muon hydrogen atoms. The muonic hydrogen atoms in the $1 s$ state had a smaller radius than that in the excited states and were therefore considered to be less susceptible to steric hindrance by the molecules.

In the liquid system, all the pion transfers occurred from the excited pionic hydrogen atoms, whereas only approximately $70 \%$ of the muon transfers occurred from the excited muonic hydrogen atoms. Meanwhile, in the gas system, no muon transfers occurred from the excited muonic hydrogen atoms. The chemical effect on the transfer reaction was significant in the order of pion transfers in the liquid system, muon transfers in the liquid system, and muon transfers in the gas system. In other words, the larger the proportion of excited states in the muonic or pionic hydrogen atoms when it transfers muons or pions, the more significant is the effect from the steric hindrance of the molecules, and the larger is the difference in the transfer rates. This indicates that the excited states of the muonic and pionic hydrogen atoms contribute significantly to the chemical effects on the transfer reaction.

Acknowledgments: This study was supported by JSPS KAKENHI Grant Number JP18K11922. The muon beam experiment at the MuSIC-M1 beamline in RCNP was conducted as an E529 experiment in the RCNP proposal number.

Author contributions: All the authors have accepted responsibility for the entire content of this submitted manuscript and approved submission.

Research funding: None declared.

Conflict of interest statement: The authors declare no conflicts of interest regarding this article.

\section{References}

1. Kubo M. K. The positive muon as a chemical and magnetic probe of metal complexes and the negative muon as a unique tool for elemental analysis. Anal. Sci. 2002, 17, i653.

2. Kubo M. K., Moriyama H., Tsuruoka Y., Sakamoto S., Koseto E., Saito T., Nishiyama K. Non-destructive elemental depth-profiling with muonic X-rays. J. Radioanal. Nucl. Chem. 2008, 278, 777.

3. Ninomiya K., Nagatomo T., Kubo K., Ito T. U., Higemoto W., Kita M., Shinohara A., Strasser P., Kawamura N., Shimomura K., Miyake Y., Saito T. Development of nondestructive and quantitative elemental analysis method using calibration curve between muonic X-ray intensity and elemental composition in bronze. Bull. Chem. Soc. Jpn. 2012, 85, 228.

4. Terada K., Ninomiya K., Osawa T., Tachibana S., Miyake Y., Kubo M. K., Kawamura N., Higemoto W., Tsuchiyama A., Ebihara M., Uesugi M. A new X-ray fluorescence spectroscopy for extraterrestrial materials using a muon beam. Sci. Rep. 2014, 4, 5072.

5. Ninomiya K., Kubo M. K., Nagatomo T., Higemoto W., Ito T. U., Kawamura N., Strasser P., Shimomura K., Miyake Y., Suzuki T., Kobayashi Y., Sakamoto S., Shinohara A., Saito T. Nondestructive elemental depth-profiling analysis by muonic X-ray measurement. Anal. Chem. 2015, 87, 4597.

6. Ninomiya K., Inagaki M., Kubo M. K., Nagatomo T., Higemoto W., Kawamura N., Strasser P., Shimomura K., Miyake Y., Sakamoto S., Shinohara A., Saito T. Negative muon induced elemental analysis by muonic X-ray and prompt gamma-ray measurements. J. Radioanal. Nucl. Chem. 2016, 309, 65.

7. Kubo M. K. Non-destructive elemental analysis using negative muon. J. Phys. Soc. Jpn. 2016, 85, 091015.

8. Terada K., Sato A., Ninomiya K., Kawashima Y., Shimomura K., Yoshida G., Kawai Y., Osawa T., Tachibana S. Non-destructive elemental analysis of a carbonaceous chondrite with direct current muon beam at MuSIC. Sci. Rep. 2017, 7, 15478.

9. Ninomiya K., Kudo T., Strasser P., Terada K., Kawai Y., Tampo M., Miyake Y., Shinohara A., Kubo K. M. Development of nondestructive isotopic analysis methods using muon beams and their application to the analysis of lead. J. Radioanal. Nucl. Chem. 2019, 320, 801.

10. Kudo T., Ninomiya K., Strasser P., Terada K., Kawai Y., Tampo M., Miyake Y., Shinohara A., Kubo K. M. Development of a nondestructive isotopic analysis method by gamma-ray emission measurement after negative muon irradiation. J. Radioanal. Nucl. Chem. 2019, 322, 1299.

11. Ninomiya K. Non-destructive, position-selective, and multielemental analysis method involving negative muons. J. Nucl. Radiochem. Sci. 2019, 19, 8.

12. Umegaki I., Higuchi Y., Kondo Y., Ninomiya K., Takeshita S., Tampo M., Nakano H., Oka H., Sugiyama J., Kubo M. K., Miyake Y. Nondestructive high-sensitivity detections of metallic lithium deposited on a battery anode using muonic X-rays. Anal. Chem. 2020, 92, 8194.

13. Sekine T., Hashimoto K., Kaji H., Yoshihara K., Imanishi N., Yoshimura Y. Pionic X-ray intensity ratios in chromium compounds. J. Radioanal. Nucl. Chem. 1989, 135, 207.

14. Placci A., Zavattini E., Bertin A., Vitale A. Direct measurement of the transfer rates of muons from $\mu p$ muonic atoms to argon, krypton and xenon atoms. Il Nuovo Cimento A 1969, 64, 1053. 
15. Baijal J. S., Diaz J. A., Kaplan S. N., Pyle R. V. Atomic capture of M-mesons in chemical compounds. Il Nuovo Cimento 1963, 30, 711.

16. Schneuwly H., Dubler T., Kaeser K., Robert-Tissot B., Schaller L. A., Schellenberg L. On the influence of the chemical bond on the relative muonic capture rates in elements of compounds. Phys. Lett. A 1978, 66, 188.

17. Yoshida G., Ninomiya K., Ito T. U., Higemoto W., Nagatomo T., Strasser P., Kawamura N., Shimomura K., Miyake Y., Miura T., Kubo K. M., Shinohara A. Muon capture probability of carbon and oxygen for $\mathrm{CO}, \mathrm{CO}_{2}$, and $\mathrm{COS}$ under low-pressure gas conditions. J. Radioanal. Nucl. Chem. 2015, 303, 1277.

18. Ninomiya K., Kitanaka M., Shinohara A., Tampo M., Miyake Y., Sakai Y., Kubo M. K. Muonic X-ray measurements on mixtures of $\mathrm{CaO} / \mathrm{MgO}$ and $\mathrm{Fe}_{3} \mathrm{O}_{4} / \mathrm{MnO}$. J. Radioanal. Nucl. Chem. 2018, 316, 1107.

19. Ninomiya K., Ito T. U., Higemoto W., Kawamura N., Strasser P., Nagatomo T., Shimomura K., Miyake Y., Kita M., Shinohara A., Kubo K. M., Miura T. Negative muon capture ratios for nitrogen oxide molecules. J. Radioanal. Nucl. Chem. 2019, 319, 767.

20. Yoshida G., Ninomiya K., Inagaki M., Higemoto W., Strasser P., Kawamura N., Shimomura K., Miyake Y., Miura T., Kubo K. M., Shinohara A. Initial quantum levels of captured muons in CO, $\mathrm{CO}_{2}$, and COS. J. Radioanal. Nucl. Chem. 2019, 320, 283.

21. Ninomiya K., Kajino M., Inagaki M., Terada K., Sato A., Tomono D., Kawashima Y., Shinohara A. Per atom muon capture ratios and effects of molecular structure on muon capture by $\mathrm{Y}-\mathrm{Fe}_{2} \mathrm{O}_{3}$ and $\mathrm{Fe}_{3} \mathrm{O}_{4}$. J. Radioanal. Nucl. Chem. 2020, 324, 403.

22. Brandão d'Oliveira A., Daniel H., von Egidy T. Coulomb capture and x-ray cascades of muons in metal halides. Phys. Rev. A 1976, 13, 1772.

23. Schneuwly H., Boschung M., Kaeser K., Piller G., Rüetschi A., Schaller L. A., Schellenberg L. Capture of negative muons in cubic and hexagonal structures of carbon and boron nitride. Phys. Rev. A 1983, 27, 950.

24. Ponomarev L. I. Molecular structure effects on atomic and nuclear capture of mesons. Annu. Rev. Nucl. Sci. 1973, 23, 395.

25. Jackson D. F., Lewis C. A., O’Leary K. Pion-capture probabilities in organic molecules. Phys. Rev. A 1982, 25, 3262.

26. Measday D. F. The nuclear physics of muon capture. Phys. Rep. 2001, 354, 243.

27. Horváth D., Bannikov A. V., Kachalkin A. K., Lévay B., Petrukhin V. I., Vasilyev V. A., Yutlandov I. A., Strakovsky I. I. Temperature breaking of hydrogen bonds in ammonia studied by $\pi^{-}$-meson capture in hydrogen. Chem. Phys. Lett. 1982, 87, 504.

28. Horváth D., Measday D. F., Entezami F., Hasinoff M. D., Noble A. J., Stanislaus S., Virtue C. J., Clough A. S., Smith J. R. H., Salomon M., Aniol K. A. Pion capture and hydrogen bonds in deuterated methanol. Phys. Rev. A 1991, 44, 1725.

29. Shinohara A., Muroyama T., Shintai J., Kurachi J., Furukawa M., Miura T., Yoshimura Y., Saito T., Ohdaira T., Imanishi N. Negativepion capture process and its chemical effects in some hydrocarbons. Phys. Rev. A 1996, 53, 130.

30. Muroyama T., Shinohara A., Saito T., Yokoyama A., Miura T., Furukawa $M$. Intensity patterns of pionic $X$ rays emitted from some organic compounds. Radiochim. Acta 1998, 80, 31.

31. Shinohara A., Muroyama T., Miura T., Saito T., Yokoyama A., Furukawa M. Behavior of pionic hydrogen atoms in liquid organic compounds. Hyperfine Interact 1997, 106, 301.

32. Thalmann Y.-A., Jacot-Guillarmod R., Mulhauser F., Schaller L. A., Schellenberg L., Schneuwly H., Tresch S., Werthmüller A. Muon transfer from excited states of hydrogen and deuterium to nitrogen, neon, and argon. Phys. Rev. A 1998, 57, 1713.

33. Yoshida G., Ninomiya K., Inagaki M., Higemoto W., Kawamura N., Shimomura K., Miyake Y., Miura T., Kubo M. K., Shinohara A. Chemical environmental effects on muon transfer process in low pressure mixture gases; $\mathrm{H}_{2}+\mathrm{CO}$ and $\mathrm{H}_{2}+\mathrm{CO}_{2}$. Radioisotopes 2016, $65,113$.

34. Inagaki M., Ninomiya K., Yoshida G., Higemoto W., Kawamura N., Miyake Y., Miura T., Shinohara A. Muon transfer rates from muonic hydrogen atoms to gaseous benzene and cyclohexane. J. Nucl. Radiochem. Sci. 2018, 18, 5.

35. Hirayama H., Namito Y., Bielajew A. F., Wilderman S. J., Nelson W. R. The EGS5 Code System. SLAC-Report-730; Stanford Linear Accelerator Center: Stanford, USA, 2005.

36. Muroyama T. Formation and Transfer Process of Pionic Hydrogen Atoms in Lquid Organic Compounds [Yuki ekitai kagobutsu ni okeru pai chukanshi suiso genshi no keisei to ten'i katei]. Ph.D. Thesis; Nagoya University: Nagoya, Japan, 1997.

37. Jacot-Guillarmod R., Mulhauser F., Piller C., Schaller L. A., Schellenberg L., Schneuwly H., Thalmann Y.-A., Tresch S., Werthmüller A., Adamczak A. Muon transfer from thermalized muonic hydrogen isotopes to argon. Phys. Rev. A 1997, 55, 3447.

38. Pisano V., Puddu G., Quarati P., Sulis L. The use of the master equation in the cascade of exotic systems in a pure Coulomb field. Il Nuovo Cimento A 1982, 72, 27. 